\title{
INFLUÊNCIA DA SUPLEMENTAÇÃO DE SAIS SOBRE O DESEMPENHO DE UM AnSBBR PARA TRATAMENTO DE SORO DE QUEIJO
}

\author{
R. A. Bezerra Jr ${ }^{1}$, A. A. de Favari ${ }^{1}$ e J. A. D. Rodrigues ${ }^{2}$ \\ ${ }^{1}$ Centro Universitário Fundação Santo André, Departamento de Engenharia Ambiental \\ ${ }^{2}$ Instituto Mauá de Tecnologia, Departamento de Engenharia Química e Alimentos \\ E-mail para contato: robertobezerrajr@yahoo.com.br
}

\begin{abstract}
RESUMO - Neste trabalho avaliou-se o desempenho de um reator anaeróbio operado em batelada seqüencial e contendo biomassa imobilizada (Anaerobic Sequencing Batch Biofilm Reactor - AnSBBR) em espuma de poliuretano. Com a finalidade de contribuir para a resolução dos problemas de aspecto tecnológico que esse tipo de sistema ainda apresenta, como controle de alcalinidade e concentração de sais no tratamento de efluentes com alta biodegradabilidade, o presente trabalho teve como objetivo principal a avaliação da influência da suplementação de sais sobre a eficiência e a estabilidade do reator para o tratamento de soro de queijo, no intuito de colaborar para a correta operação deste tipo de reator. Os resultados mostraram um aumento nos valores de eficiência de remoção de matéria orgânica, ou seja, um aumento no desempenho do reator nas condições em que houve a suplementação de sais no afluente; no entanto, verificou-se que a concentração desses sais não influenciou o desempenho do reator. Os valores de eficiência de remoção de matéria orgânica para a primeira condição, sem adição de sais, ficaram em torno de $81 \%$ e $85 \%$, para amostras não filtradas e filtradas do efluente, respectivamente. Para as condições com adição de sais, estes valores subiram para $89 \%$ e $92 \%$, respectivamente. Além disso, foi verificado que não houve acúmulo de ácidos, o reator manteve sua estabilidade em todas as condições estudadas, mantendo o $\mathrm{pH}$ do meio próximo da neutralidade.
\end{abstract}

\section{INTRODUÇÃO}

Devido à sua flexibilidade operacional, o reator anaeróbio operado em batelada sequencial tem sido amplamente utilizado para o tratamento de diversos tipos de águas residuárias (Massé e Masse, 2000; Angenent et al., 2001; Cubas et al., 2004; Zaiat et al., 2001; Camargo et al., 2002; Orra et al., 2004; Ramos et al., 2003; Chebel et al., 2006; Damasceno et al., 2007; Bezerra et al., 2009; Bezerra et al., 2011; Lovato et al., 2012). A disposição do soro de queijo em corpos d'água pode ser minimizada com o tratamento biológico anaeróbio deste tipo de efluente em reator de batelada, reduzindo seu potencial poluidor e, ao mesmo tempo, gerando metano, que pode ser aproveitado na própria indústria como fonte de energia. Entretanto, a altíssima biodegradabilidade, a alta carga orgânica, a deficiência de alcalinidade e a ausência de micronutrientes e de um sistema de controle do $\mathrm{pH}$ fazem com que o tratamento anaeróbio desse tipo de substrato em reatores de alta taxa, embora possível, se torne difícil (Boening e Larsen, 1982; Kelly e Switzenbaum, 1984; Lo e Liao, 1986; Malaspina et al., 1995). Por causa desta elevada biodegradabilidade, o soro tende a acidificar-se rapidamente, dificultando a estabilidade do processo. Em grande parte dos casos, torna-se necessária então a adição de alguma fonte externa de alcalinidade, na forma de 
bicarbonato, carbonato ou algum hidróxido (Yan et al., 1992), bem como a adição de alguns sais. Além disso, de acordo com Omil et al. (2003), a adição de sais e de fonte externa de alcalinidade não somente aumenta a capacidade tampão do meio, como também facilita a degradação anaeróbia de lipídeos presentes no soro. Sendo assim, com a finalidade de contribuir para a resolução desses problemas de aspecto tecnológico que esse tipo de sistema ainda apresenta, no tratamento de soro de queijo, o presente trabalho teve como objetivo principal a avaliação da influência da suplementação de sais sobre a eficiência e a estabilidade de um AnSBBR para o tratamento de soro de queijo, no intuito de colaborar para a correta operação deste tipo de reator.

\section{MATERIAIS E MÉTODOS}

O reator utilizado neste trabalho foi inicialmente proposto por Ratusznei et al. (2001), sendo constituído por um frasco de acrílico, cilíndrico, de $150 \mathrm{~mm}$ de diâmetro e $155 \mathrm{~mm}$ de altura, com capacidade total nominal de 2,0 L. A mistura do meio reacional foi promovida por agitador magnético (Marconi, modelo MA 085). Para manter sua temperatura aproximadamente constante, o reator foi colocado numa incubadora (Marconi, modelo MA $415 / \mathrm{S}$ ) e mantido a $30 \pm 1^{\circ} \mathrm{C}$. A concentração de matéria orgânica no afluente foi de, aproximadamente, $500 \mathrm{mgDQO} / \mathrm{L}$. O tempo de ciclo foi de 24 horas, e o volume de soro de queijo tratado a cada ciclo foi de 1,3 L. A alimentação foi realizada por um funil de vidro e uma mangueira, que ficava dentro do reator, e a descarga do reator foi realizada utilizando a mesma mangueira de alimentação e um pipetador de borracha para sucção da água residuária. Como suporte de imobilização da biomassa, foi utilizada espuma de poliuretano na forma de cubos de $1,0 \mathrm{~cm}$ de aresta. As biopartículas foram confinadas em um cesto de plástico perfurado de $110 \mathrm{~mm}$ de altura, possuindo diâmetro de $140 \mathrm{~mm}$. As partes laterais do cilindro interno, superior e inferior do cesto continham uma tela de modo a confinar o suporte contendo a biomassa imobilizada, utilizando a própria parede do reator como limite externo do cesto, não necessitando, assim, de tela. $\mathrm{O}$ inóculo foi proveniente de reator anaeróbio de manta de lodo e escoamento ascendente, tratando efluente de abatedouro de aves. A água residuária sintética foi preparada dissolvendo-se soro de queijo desidratado em água de torneira, sendo que, experimentalmente, $500 \mathrm{mg}$ de soro desidratado correspondeu à, aproximadamente, $500 \mathrm{mg}$ de DQO. Além de soro de queijo, o afluente do reator foi suplementado com bicarbonato de sódio, na razão de uma grama de bicarbonato de sódio para cada grama de DQO alimentada ao reator e, em algumas condições operacionais, suplementado com alguns sais. O estudo da influência da suplementação de sais sobre a eficiência e a estabilidade do processo foi realizado operando-se o reator com concentrações crescentes de sais no afluente. Ao todo foram estudadas quatro condições operacionais. $\mathrm{Na}$ primeira condição operacional, o afluente foi composto apenas por soro de queijo e bicarbonato de sódio. Nas condições operacionais seguintes, variou-se a concentração de sais no afluente, mantendo-se a mesma concentração de soro de queijo e bicarbonato de sódio em todas as condições. Na Tabela 1 é apresentado os valores da concentração de cada sal utilizado nas quatro condições operacionais investigadas. As variáveis monitoradas durante a operação do reator foram: concentração de matéria orgânica nas formas não filtrada $\left(\mathrm{C}_{\mathrm{ST}}\right) \mathrm{e}$ filtrada $\left(\mathrm{C}_{\mathrm{SF}}\right)$, alcalinidade a bicarbonato $(\mathrm{AB})$, ácidos voláteis totais (AVT), sólidos totais (ST), sólidos totais voláteis (STV), sólidos suspensos totais (SST) e sólidos suspensos voláteis (SSV), além da medida do $\mathrm{pH}$ e do volume de meio descarregado. As análises foram realizadas de acordo com o Standard Methods for the Examination of Water and Wastewater (1995). Ao final de cada condição foram retiradas do reator amostras de biopartículas para (1) análise microbiológica, a qual foi realizada por microscopia óptica comum e de contraste de fase por fluorescência, utilizando microscópio Olympus ${ }^{\circledR}$ modelo BX41, e para (2) a análise 
das concentrações de sólidos totais $\left(\mathrm{S}_{\mathrm{ST}}\right)$ e sólidos totais voláteis $\left(\mathrm{S}_{\mathrm{STV}}\right)$ presentes no reator, podendo-se ter, assim, uma estimativa da concentração de biomassa no reator, na forma de miligramas de sólidos por grama de espuma seca (mg sólidos/g espuma seca), e na forma de gramas de sólidos por litro de meio reacional no reator (g sólidos/L meio reacional).

Tabela 1 - Concentração de sais no afluente do reator para cada condição estudada

\begin{tabular}{cccc}
\hline Condição & $\mathrm{NaCl}(\mathrm{mg} / \mathrm{L})$ & $\mathrm{CaCl}_{2} \cdot 2 \mathrm{H}_{2} \mathrm{O}(\mathrm{mg} / \mathrm{L})$ & $\mathrm{MgCl}_{2} \cdot 6 \mathrm{H}_{2} \mathrm{O}(\mathrm{mg} / \mathrm{L})$ \\
\hline I & - & - & - \\
II & 76,0 & 1,35 & 2,10 \\
III & 152 & 2,70 & 4,20 \\
IV & 228 & 4,05 & 6,30 \\
\hline
\end{tabular}

\section{RESULTADOS E DISCUSSÃO}

A Tabela 2 apresenta os valores médios de concentração de matéria orgânica para amostras não filtradas $\left(\mathrm{C}_{\mathrm{ST}}\right)$ e filtradas $\left(\mathrm{C}_{\mathrm{SF}}\right)$, eficiência de remoção de matéria orgânica para amostras não filtradas $\left(\varepsilon_{\mathrm{ST}}\right)$ e filtradas $\left(\varepsilon_{\mathrm{SF}}\right)$, concentração de ácidos voláteis totais (AVT), alcalinidade a bicarbonato $(\mathrm{AB}), \mathrm{pH}$, sólidos totais (ST), sólidos totais voláteis (STV), sólidos suspensos totais (SST), sólidos suspensos voláteis (SSV), e carga orgânica removida para amostras não filtradas $\left(\mathrm{COR}_{\mathrm{ST}}\right)$ e filtradas $\left(\mathrm{COR}_{\mathrm{SF}}\right)$, ao longo de todo o período de operação do reator, para as quatro condições operacionais estudadas. As Figuras 1 e 2 ilustram, respectivamente, os valores de concentração de matéria orgânica no efluente e de eficiência de remoção de matéria orgânica, ao longo de todo o período de operação do reator. O reator foi operado durante um período total de 58 dias. O tempo de operação da primeira, segunda, terceira e quarta condição operacional, foi de 16,14, 18 e 10 dias. Como se pode observar na Figura 1, durante os primeiros sete dias de operação, o reator apresentou valores ainda altos de concentração de matéria orgânica, tanto para amostras filtradas quanto para amostras não filtradas do efluente, porém, valores estes já menores do que aqueles apresentados pelo afluente (média de $508 \mathrm{mgDQO} / \mathrm{L}$ ). A partir do nono dia de operação, já verificou-se uma estabilização nos valores das variáveis monitoradas. A Tabela 2 mostra que para a primeira condição operacional estudada, os valores de concentração de matéria orgânica para amostras filtradas e não filtradas do efluente ficaram em torno de, respectivamente, 76 e $95 \mathrm{mgDQO} / \mathrm{L}$, e os valores de eficiência de remoção de matéria orgânica, em torno de $85 \%$ e $81 \%$, respectivamente. Na segunda condição, foi verificado uma queda na concentração de matéria orgânica no efluente em relação à primeira condição, tanto para amostras filtradas (39 mgDQO/L) quanto para amostras não filtradas (54 mgDQO/L) do efluente, o que resultou em valores de eficiência de remoção de matéria orgânica maiores, tanto para amostras filtradas $(92 \%)$ quanto para amostras não filtradas $(89 \%)$ do efluente, em relação àqueles verificados na primeira condição. Isto mostra que a adição de sais ao afluente resultou em uma melhoria na qualidade do efluente, ou seja, em menores valores de concentração de matéria orgânica no efluente e, portanto, em maiores valores de eficiência. Na terceira e quarta condição operacional, foram verificados valores de concentração de matéria orgânica no efluente e eficiência de remoção de matéria orgânica muito próximos daqueles observados na segunda condição operacional, ou seja, embora a presença de sais no meio tenha aumentado a eficiência do reator, a quantidade desses sais no meio não influenciou os valores de eficiência. A análise da Figura 1 ilustra mais claramente a queda nos valores de concentração de matéria orgânica no efluente, tanto para amostras filtradas quanto para não filtradas, durante a passagem da primeira para segunda condição operacional, em que já houve a adição de sais. A Figura 1 também mostra que a concentração de matéria orgânica no efluente manteve-se praticamente constante para a segunda, terceira e quarta condição, 
comprovando que apenas a presença, e não a quantidade de sais, influenciou o desempenho do reator. Consequentemente, a análise da Figura 2 mostrou um aumento nos valores de eficiência de remoção de matéria orgânica, tanto para amostras filtradas quanto para amostras não filtradas do efluente, quando da presença de sais no afluente, com manutenção dos valores de eficiência para segunda, terceira e quarta condições estudadas.

Tabela 2 - Valores médios das variáveis monitoradas para as quatro condições estudadas *

\begin{tabular}{|c|c|c|c|c|c|}
\hline \multirow{2}{*}{ Variável } & \multirow{2}{*}{ Afluente } & \multicolumn{4}{|c|}{ Efluente } \\
\hline & & Condição I & Condição II & Condição III & Condição IV \\
\hline $\begin{array}{c}\mathrm{C}_{\mathrm{ST}} \\
(\mathrm{mgDQO} / \mathrm{L})\end{array}$ & $508 \pm 13^{(24)}$ & $95 \pm 10^{(6)}$ & $54 \pm 6^{(6)}$ & $55 \pm 1^{(9)}$ & $56 \pm 2^{(7)}$ \\
\hline $\begin{array}{l}\varepsilon_{\mathrm{ST}} \\
(\%)\end{array}$ & - & $81 \pm 2^{(6)}$ & $89 \pm 1^{(6)}$ & $89 \pm 0^{(9)}$ & $89 \pm 0^{(7)}$ \\
\hline $\begin{array}{c}\mathrm{C}_{\mathrm{SF}} \\
(\mathrm{mgDQO} / \mathrm{L})\end{array}$ & - & $76 \pm 11^{(6)}$ & $39 \pm 9^{(6)}$ & $36 \pm 2^{(9)}$ & $37 \pm 1^{(7)}$ \\
\hline $\begin{array}{l}\varepsilon_{\mathrm{SF}} \\
(\%)\end{array}$ & - & $85 \pm 2^{(6)}$ & $92 \pm 2^{(6)}$ & $93 \pm 0^{(9)}$ & $93 \pm 0^{(7)}$ \\
\hline $\begin{array}{c}\mathrm{AVT} \\
(\mathrm{mgHAc} / \mathrm{L})\end{array}$ & $53 \pm 8^{(24)}$ & $65 \pm 6^{(7)}$ & $52 \pm 4^{(6)}$ & $51 \pm 5^{(9)}$ & $47 \pm 2^{(7)}$ \\
\hline $\begin{array}{c}\mathrm{AB} \\
\left(\mathrm{mgCaCO}_{3} / \mathrm{L}\right)\end{array}$ & $300 \pm 14^{(24)}$ & $370 \pm 62^{(7)}$ & $330 \pm 41^{(6)}$ & $324 \pm 67^{(9)}$ & $317 \pm 16^{(7)}$ \\
\hline $\mathrm{pH}$ & $7,6 \pm 0,3^{(24)}$ & $7,8 \pm 0,2^{(7)}$ & $7,9 \pm 0,2^{(6)}$ & $7,8 \pm 0,5^{(9)}$ & $8,0 \pm 0,4^{(7)}$ \\
\hline $\begin{array}{c}\mathrm{ST} \\
(\mathrm{mg} / \mathrm{L})\end{array}$ & $1152 \pm 58^{(15)}$ & $776 \pm 47^{(4)}$ & $895 \pm 39^{(3)}$ & $712 \pm 42^{(5)}$ & $803 \pm 45^{(3)}$ \\
\hline $\begin{array}{c}\text { STV } \\
(\mathrm{mg} / \mathrm{L})\end{array}$ & $861 \pm 39^{(15)}$ & $395 \pm 35^{(4)}$ & $487 \pm 58^{(3)}$ & $451 \pm 54^{(5)}$ & $409 \pm 34^{(3)}$ \\
\hline $\begin{array}{c}\text { SST } \\
(\mathrm{mg} / \mathrm{L})\end{array}$ & $43 \pm 18^{(15)}$ & $31 \pm 14^{(4)}$ & $35 \pm 12^{(3)}$ & $30 \pm 11^{(5)}$ & $28 \pm 18^{(3)}$ \\
\hline $\begin{array}{c}\mathrm{SSV} \\
(\mathrm{mg} / \mathrm{L})\end{array}$ & $29 \pm 12^{(15)}$ & $25 \pm 11^{(4)}$ & $22 \pm 14^{(3)}$ & $26 \pm 12^{(5)}$ & $28 \pm 9^{(3)}$ \\
\hline $\begin{array}{c}\mathrm{COR}_{\mathrm{ST}} \\
(\mathrm{mgDQO} / \mathrm{L} . \mathrm{d})\end{array}$ & - & $318 \pm 8^{(6)}$ & $348 \pm 4^{(6)}$ & $348 \pm 1^{(9)}$ & $347 \pm 1^{(7)}$ \\
\hline $\begin{array}{c}\mathrm{COR}_{\mathrm{SF}} \\
(\mathrm{mgDQO} / \mathrm{L} \cdot \mathrm{d})\end{array}$ & - & $332 \pm 9^{(6)}$ & $361 \pm 7^{(6)}$ & $361 \pm 7^{(9)}$ & $362 \pm 1^{(7)}$ \\
\hline
\end{tabular}

* Entre parênteses têm-se o número de amostras consideradas para o cálculo da média.

Biomassa presente no reator (valor médio para as quatro condições operacionais):

$\mathrm{S}_{\mathrm{ST}}=2157 \mathrm{mg}$ sólidos totais $/ \mathrm{g}$ espuma seca; $\mathrm{S}_{\mathrm{STV}}=1769 \mathrm{mg}$ sólidos totais voláteis $/ \mathrm{g}$ espuma seca;

$\mathrm{S}_{\mathrm{ST}}=41,6 \mathrm{~g}$ sólidos totais/L meio reacional; $\mathrm{S}_{\mathrm{STV}}=34,1 \mathrm{~g}$ sólidos totais voláteis/L meio reacional;

$\mathrm{S}_{\mathrm{STV}} / \mathrm{S}_{\mathrm{ST}}=0,82 \mathrm{~g}$ sólidos totais voláteis $/ \mathrm{g}$ sólidos totais. 


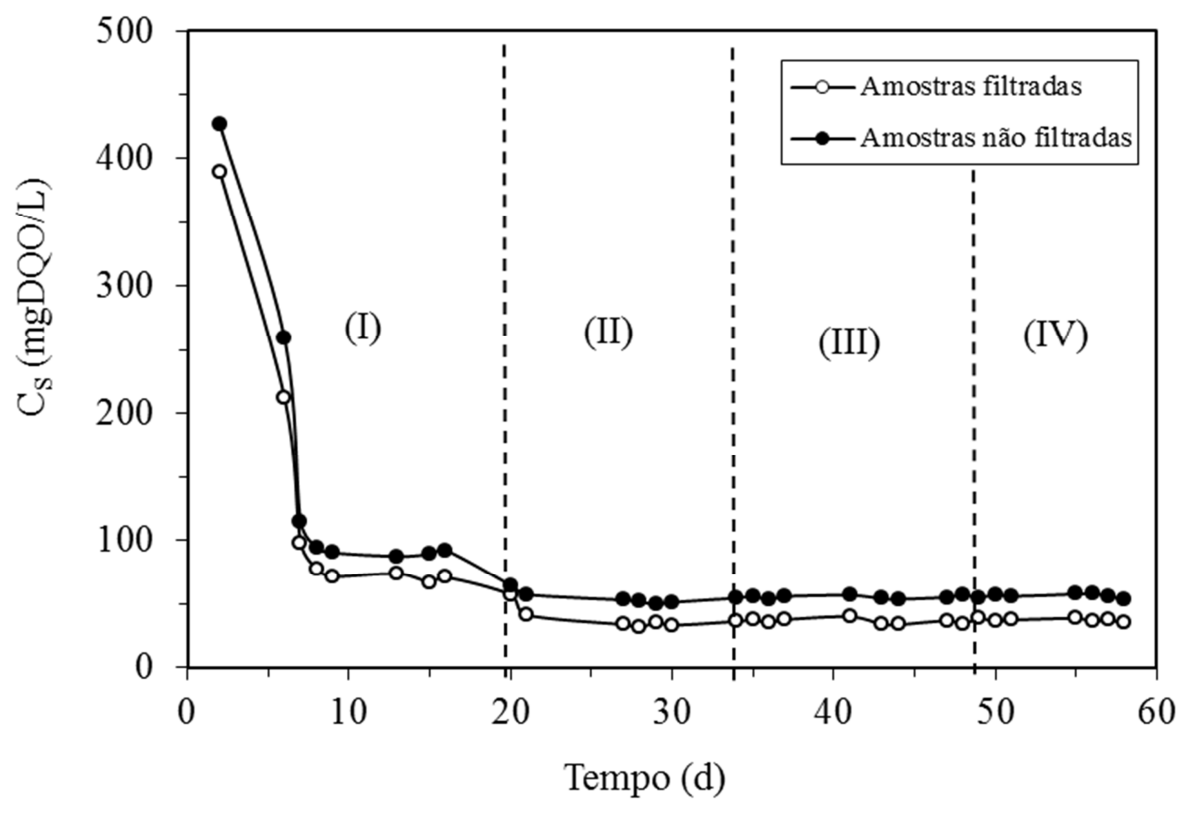

Figura 1 - Concentração de matéria orgânica no efluente do reator durante todo o período de operação.

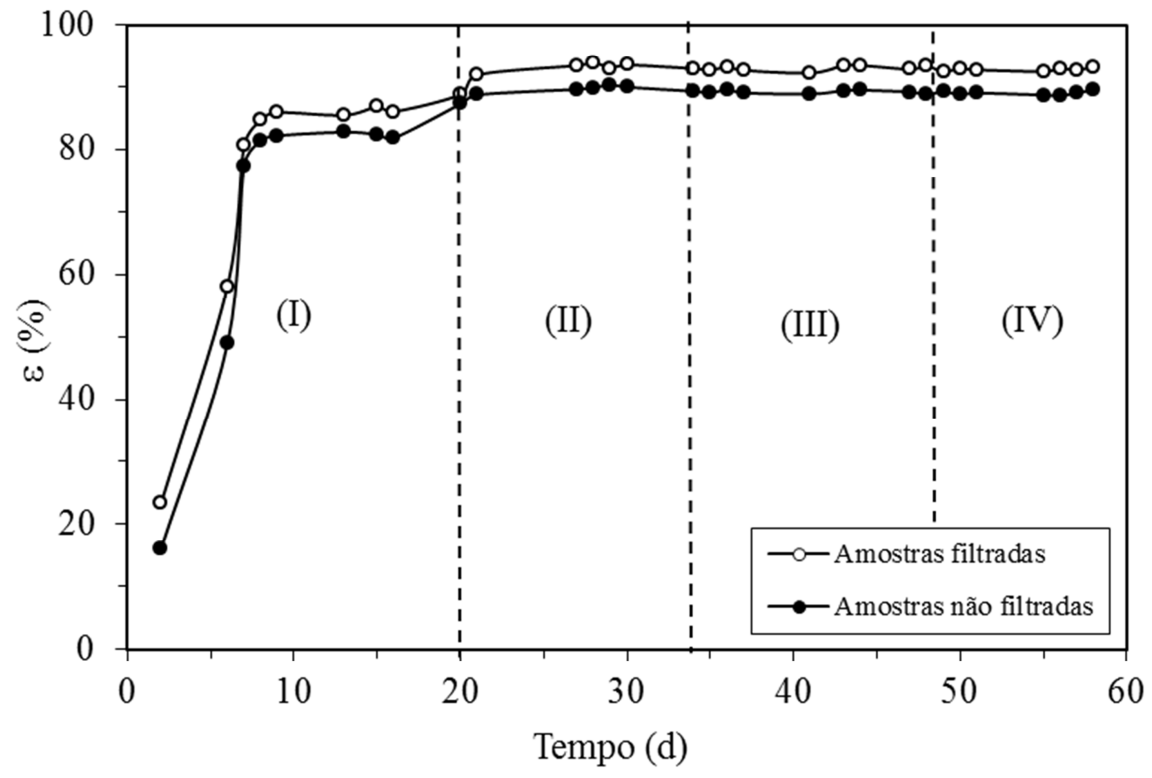

Figura 2 - Eficiência de remoção de matéria orgânica pelo reator durante todo o período de operação.

Quantos aos valores de concentração de ácidos voláteis totais no efluente, estes seguiram a mesma tendência dos valores de concentração de matéria orgânica, ou seja, apresentaram uma queda nas condições em que houve suplementação de sais no afluente. Conforme mostrado na Tabela 2, para a primeira condição operacional, em que não houve suplementação de sais, os valores de concentração de ácidos voláteis totais no efluente permaneceram em torno de $65 \mathrm{mgHAc} / \mathrm{L}$, enquanto para as demais condições operacionais em que houve suplementação de sais no afluente, aqueles valores ficaram em torno de $50 \mathrm{mgHAc} / \mathrm{L}$, comprovando que a presença de sais no meio também colaborou para minimizar a quantidade de ácidos voláteis totais no efluente do reator. 
Com relação aos valores médios de alcalinidade a bicarbonato no efluente, verificou-se que estes permaneceram maiores do que aqueles no afluente em todas as condições estudadas, comprovando que o sistema produziu alcalinidade, mantendo o $\mathrm{pH}$ do meio próximo do valor neutro em todas as condições, e colaborando portanto para o auto controle do processo anaeróbio entre a produção e o consumo de ácidos no reator. Com relação aos valores de concentração de sólidos totais, totais voláteis, suspensos totais e suspensos voláteis no efluente, estes permaneceram menores do que aqueles verificados no afluente, comprovando também a capacidade de remoção de sólidos pelo reator em todas as condições operacionais. A Figura 3 ilustra os valores de carga orgânica removida pelo reator ao longo de todo o período de operação, para todas as condições estudadas. A análise da Figura 3 mostra que os valores de carga orgânica removida seguiram a mesma tendência dos valores de eficiência de remoção de matéria orgânica, ou seja, durante os primeiros sete dias de operação, o reator apresentou valores ainda baixos de carga orgânica removida, tanto para amostras filtradas quanto para amostras não filtradas do efluente. A partir do nono dia de operação, porém, já verificou-se uma estabilização nos valores de carga orgânica removida pelo reator, os quais permaneceram, para a primeira condição operacional, sem a presença de sais no meio, em torno de 318 e $332 \mathrm{mgDQO} / \mathrm{L} . \mathrm{d}$, para amostras não filtradas e filtradas do efluente, respectivamente. Para as demais condições operacionais, em que houve a presença de sais no meio, verificou-se um aumento nos valores de carga orgânica removida para amostras não filtradas e filtradas do efluente, que permaneceram em torno de 348 e $361 \mathrm{mgDQO} / \mathrm{L} . \mathrm{d}$, respectivamente. Estes resultados mostram um aumento do desempenho do reator (não somente do ponto de vista qualitativo, como também quantitativo) quando da presença de sais no meio, sem, contudo, que a concentração desses sais influenciasse o desempenho do reator. Isto nos leva a concluir que os sais possam ter se comportado como uma espécie de catalisador, aumentando a velocidade do consumo de matéria orgânica sem, porém, participar efetivamente da reação. Em todas as condições avaliadas, as análises microbiológicas mostraram a existência de morfologias semelhantes à Methanosarcina sp. e Methanosaeta sp., havendo um equilíbrio destes dois últimos gêneros.

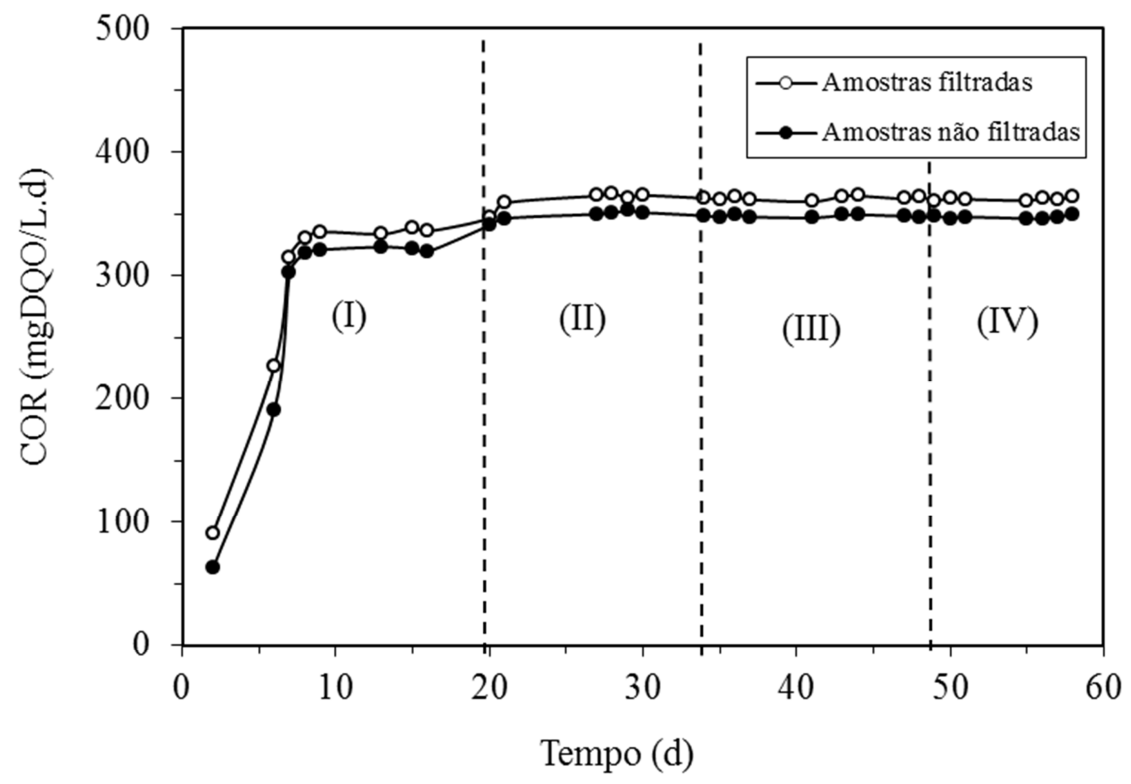

Figura 3 - Carga orgânica removida pelo reator durante todo o período de operação. 


\section{CONCLUSÕES}

Os resultados mostraram um aumento dos valores de eficiência de remoção de matéria orgânica pelo reator quando houve a adição de sais no meio, porém, a concentração de sais não influenciou o desempenho do reator em termos de eficiência. Os valores de eficiência de remoção de matéria orgânica para a primeira condição, sem adição de sais, ficaram em torno de $81 \%$ e $85 \%$, para amostras não filtradas e filtradas do efluente, respectivamente. Para as condições com adição de sais, estes valores subiram para $89 \%$ e $92 \%$, respectivamente. Os valores de carga orgânica removida seguiram a mesma tendência dos valores de eficiência, ou seja, aumentaram com a presença de sais, mas não foram influenciados pela concentração desses sais no meio. Para a primeira condição, na ausência de sais, os valores médios de carga orgânica removida, para amostras não filtradas e filtradas do efluente, ficaram em torno de, respectivamente, 318 e $332 \mathrm{mgDQO} / \mathrm{L} . \mathrm{d}$. Para as demais condições, na presença de sais no meio, aqueles valores mantiveram-se em torno de 348 e $361 \mathrm{mgDQO} / \mathrm{L} . \mathrm{d}$, respectivamente. Isso comprova que a suplementação de sais no meio aumentou o desempenho do reator não somente do ponto de vista qualitativo, mas também quantitativo, ou seja, aumentou não somente a qualidade do efluente, como também a quantidade de matéria orgânica removida pelo reator. Quanto à concentração de ácidos voláteis totais no efluente, a adição de sais no meio colaborou para minimizá-la e, portanto, para aumentar a estabilidade do reator e o auto controle entre a geração e consumo de ácidos. Em geral, concluiu-se que a adição de sais no meio aumentou o desempenho e a estabilidade do reator. Na prática, em um processo industrial anaeróbio de tratamento, uma alternativa à adição de sais seria a suplementação, no meio, do esgoto gerado pela própria indústria, uma vez que este possui alta concentração de sais e alcalinidade, reduzindo assim os custos com aquisição de sais e, ao mesmo tempo, realizando também o tratamento do esgoto gerado.

\section{REFERÊNCIAS BIBLIOGRÁFICAS}

ANGENENT, L. T.; SUNG, S.; RASKIN, L. Mixing intensity in anaerobic sequencing batch reactors affects reactor performance and microbial community structure. In: Proceedings of the $9^{\text {th }}$ World Congress on Anaerobic Digestion, Antwerpen, Belgium, p. 267-274, 2001.

BEZERRA, R. A.; RODRIGUES, J. A. D.; RATUSZNEI, S. M.; ZAIAT, M.; FORESTI, E. Effects of feed time, organic loading and shock loads in the anaerobic whey treatment by an AnSBBR with circulation. Applied Biochemistry and Biotechnology, v. 157, p. 140-158, 2009.

BEZERRA, R. A.; RODRIGUES, J. A. D.; RATUSZNEI, S. M.; CANTO, C. S. A.; ZAIAT, M. Effect of Organic Load on the Performance and Methane Production of an AnSBBR Treating Effluent from Biodiesel Production. Applied Biochemistry and Biotechnology, v. 165(01), p. 347-368, 2011.

BOENING, P. H.; LARSEN, V. F. Anaerobic fluidized bed whey treatment. Biotechonology Bioenginnering, v. 24, p. 2539-2556, 1982.

CAMARGO, E. F. M.; RATUSZNEI, S. M.; RODRIGUES, J. A. D.; ZAIAT, M.; BORZANI, W. Treatment of low-strength wastewater using immobilized biomass in a sequencing batch external loop reactor: Influence of the medium superficial velocity on the stability and performance. Brazilian Journal of Chemical Engineering, v. 19(03), p. 267-275, 2002. 
CHEBEL, F. X.; RATUSZNEI, S. M.; RODRIGUES, J. A. D.; ZAIAT, M.; FORESTI, E. Analysis of performance of an anaerobic sequential batch reactor submitted to increasing organic load with different influent concentrations and cycle lengths. Applied Biochemistry and Biotechnology, v. 133, p. 171-187, 2006.

CUBAS, S. A.; FORESTI, E.; RODRIGUES, J. A. D.; RATUSZNEI, S. M.; ZAIAT, M. Influence of liquid-phase mass transfer on the performance of a stirred anaerobic sequencing batch reactor containing immobilized biomass. Biochemical Engineering Journal, v. 17(02), p. 99-105, 2004.

DAMASCENO, L. H. S.; RODRIGUES, J. A. D.; RATUSZNEI, S. M.; ZAIAT, M.; FORESTI, E. Effects of feeding time and organic loading in na anaerobic sequencing batch biofilm reactor (ASBBR) treating diluted whey. Journal of Environmental Management, v. 85(04), p. 927-935, 2007.

KELLY, C. R.; SWITZENBAUM, M. S. Anaerobic treatment: Temperature and nutrient effects. Agricultural Waste, v. 10, p. 135-154, 1984.

LO, K. V.; LIAO, P. H. Digestion of cheese whey with anaerobic rotating biological contact reactor. Biomass, v. 10, p. 243-252, 1986.

LOVATO, G.; BEZERRA, R. A.; RODRIGUES, J. A. D.; RATUSZNEI, S. M..; ZAIAT, M. Effect of Feed Strategy on Methane Production and Performance on an AnSBBR Treating Effluent from Biodiesel Production. Applied Biochemistry and Biotechnology, v. 166, p. 2007-2029, 2012.

MALASPINA, F.; STANTE, L.; CELLAMARE, C. M.; TILCHE, A. Cheese whey and cheese factory wastewater treatment with a biological anaerobic-aerobic process. Water Science and Technology, v. 32(12), p. 59-72, 1995.

MASSÉ, D. I.; MASSE, L.Treatment of slaghterhouse wastewater in anaerobic sequencing batch reactors. Canadian Agricultural Engineering, v. 42, p. 131-137, 2000.

OMIL, F.; GARRIDO, J. M.; ARROJO, B.; MÉNDEZ, R. Anaerobic filter reactor performance for the treatment of complex dairy wastewater at industrial scale. Water Research, v. 37, p. 4099-4108, 2003.

ORRA, A. A.; RATUSZNEI, S. M.; RODRIGUES, J. A. D.; FORESTI, E.; ZAIAT, M. Effects of feeding strategies on the performance of an anaerobic discontinuous reactor containing immobilized biomass with circulation system for liquid-phase mixing. Water Science and Technology, v. 49(11-12), p. 303-310, 2004.

RAMOS, A. C. T.; RATUSZNEI, S. M.; RODRIGUES, J. A. D.; ZAIAT, M. Mass transfer improvement of a fixed-bed anaerobic sequencing batch reactor with liquid phase circulation. Journal of Science and Technology of the Americas - INTERCIENCIA, v. 28(04), p. 214-219, 2003.

RATUSZNEI, S. M.; RODRIGUES, J. A. D.; CAMARGO, E. F. M.; ZAIAT, M.; BORZANI, W. Influence of agitation rate on the performance of a stirred anaerobic sequencing batch reactor containing immobilized biomass. Water Science and Technology, v. 44(04), p. 305-412, 2001.

STANDARD METHODS FOR THE EXAMINATION OF WATER AND WASTEWATER. APHA, AWWA, WPCF. $19^{\text {th }}$ edition, American Public Health Association, Washington, 1995.

YAN, J. Q.; LO, K. V.; PINDER, K. L. Instability caused by high strength of cheese whey in a UASB reactor. Biotechnology and Bioenginnering, v. 41, p. 700-706, 1992.

ZAIAT, M.; RODRIGUES, J. A. D.; RATUSZNEI, S. M.; CAMARGO, E. F. M.; BORZANI, W. Anaerobic sequencing batch reactors for wastewater treatment: A developing technology. Applied Microbiology and Biotechnology, v. 55(01), p. 29-35, 2001. 